\title{
ENTREVISTA AL DEPARTAMENTO DE LENGUA ESPAÑOLA DE LA Dirección General de TraduCción (DGT) DE LA CoMisión EUROPEA. \\ José Luis Vega (JeFe del DePartamento); Alberto RiVAS (COORDINADOR DE CALIDAD) Y LUIS GONZÁleZ (TERMINOLÓGO). Traducción DEl IngLÉs: Cristina Álvaro Aranda
}

Por Carmen Valero-Garcés

Universidad de Alcalá carmen.valero@uah.es

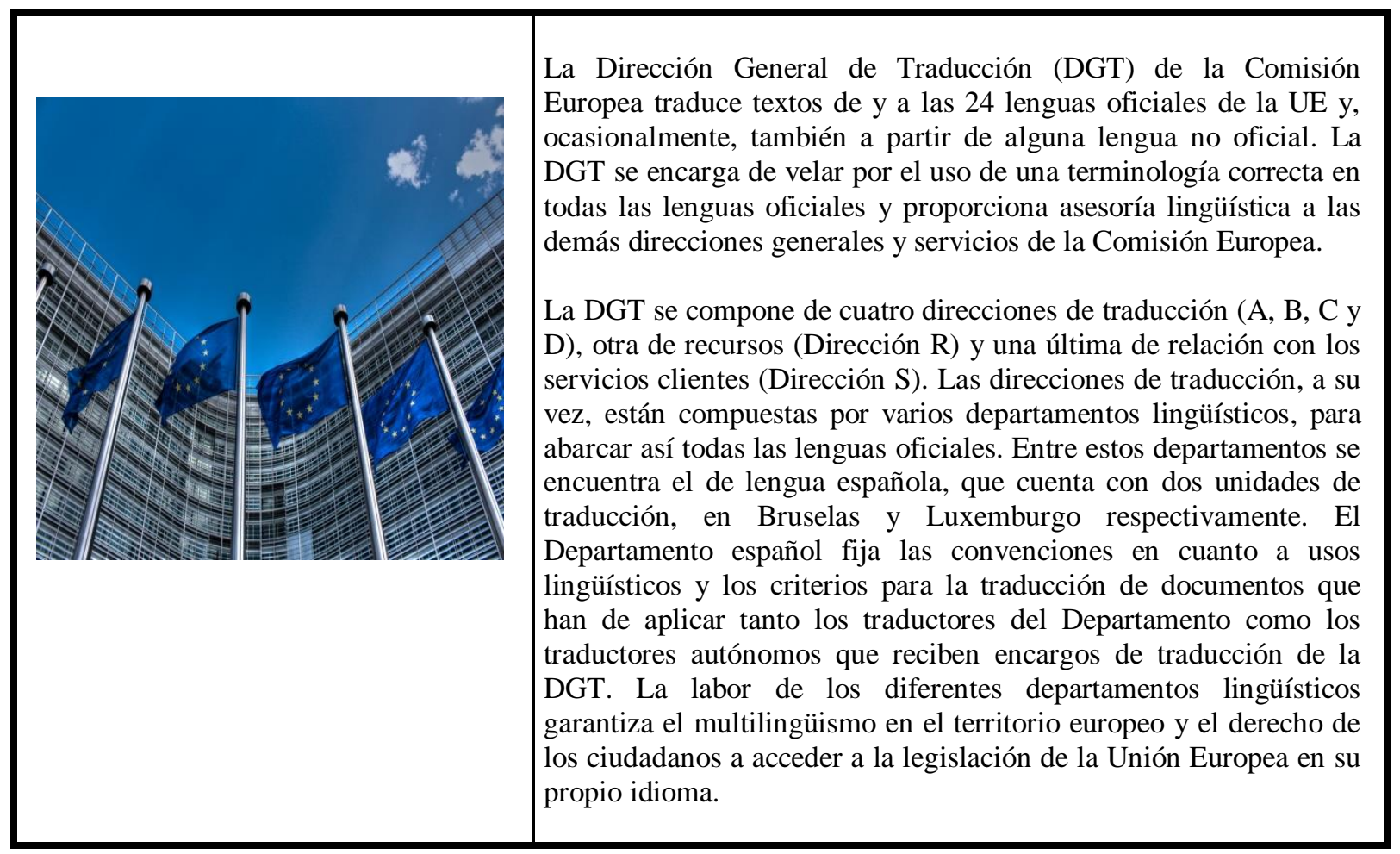

1. ¿Qué papel ocupa el uso de las nuevas tecnologías de la información y la comunicación (TICS) en la traducción en la UE?

En la Dirección General de Traducción (DGT) de la Comisión Europea utilizamos, de manera intensiva, un buen número de aplicaciones informáticas específicas. Las herramientas de traducción asistida por ordenador (TAO) y las bases de datos lingüísticas son una parte esencial del conjunto de recursos de los que se sirve la DGT (traductores de plantilla, traductores autónomos y herramientas informatizadas) y contribuyen a la prestación eficiente de servicios de alta calidad a nuestros clientes. De forma más concreta, los sistemas 
informáticos que la DGT desarrolla y mantiene se utilizan para los siguientes objetivos principales:

- Facilitar a traductores, editores y terminólogos instrumentos eficaces que respalden la precisión, consistencia y eficiencia en su trabajo, por ejemplo: herramientas de traducción asistida por ordenador, sistemas automatizados de preprocesamiento de la información y traducción automática y acceso a corpus de gran calidad, repositorios de datos y recursos terminológicos y documentales.

- Proporcionar a los gestores herramientas adecuadas para administrar el reparto de tareas y supervisar entregas.

- Ofrecer al personal administrativo herramientas eficaces que les permitan gestionar la demanda de traducción y que faciliten a la DGT la externalización de una parte de la producción para poder, de esta manera, equilibrar la oferta y la demanda.

- Facilitar a los traductores externos instrumentos adecuados para que puedan colaborar y comunicarse con la DGT.

- Proporcionar a todos los servicios de traducción de las instituciones de la Unión Europea acceso a herramientas comunes desarrolladas por la DGT, consiguiendo así economías de escala y mejorando la coherencia de las traducciones realizadas.

- Ofrecer un servicio de traducción automática a las instituciones de la Unión Europea y a las administraciones públicas, así como a los servicios en línea en los Estados miembros.

\section{2. ¿Cuáles son las herramientas más utilizadas? ¿Qué lugar ocupa la traducción automática (TA)?}

Además de instrumentos relacionados con el flujo de trabajo, los principales recursos informáticos que utilizan los traductores de la DGT siguen siendo Euramis, IATE y la herramienta de traducción asistida por ordenador (TAO) SDL Studio 2015. Recientemente hemos pasado a la última versión de nuestra herramienta de TAO, que se integra en un amplio entorno de instrumentos de este tipo. La modularidad, la interoperabilidad y la flexibilidad son conceptos esenciales en el desarrollo de nuestras tecnologías de la información.

Euramis (Sistema Europeo Avanzado de Información Plurilingüe) es un conjunto integrado de bases de datos o memorias (con sus herramientas asociadas) que gestionan las instituciones de la Unión Europea de manera colectiva o individual. Más que de una memoria, se trata de un repositorio multilingüe de fragmentos equivalentes de textos y frases (segmentos) que pertenecen a documentos oficiales de la Unión y permiten su reutilización en la traducción realizada en todas las instituciones europeas. Asimismo, el sistema dispone de un conjunto de interfaces para administrar los derechos de acceso a la gestión de frases y documentos, basados en la identificación del usuario al inicio de la sesión, tanto si se trata de personal de la Comisión como de otras instituciones de la UE. Este repositorio de memorias de traducción compartidas contiene la mayoría (pero no todos ellos) de los documentos que constituyen el acervo comunitario y también otros que no forman parte de él.

IATE (Terminología Interactiva para Europa) es la base de datos terminológica interinstitucional de la Unión Europea. Se utiliza en las instituciones y agencias europeas desde el verano de 2004 con el objetivo de recopilar, difundir y gestionar de forma 
compartida la terminología específica de la Unión. Más adelante nos detendremos algo más en este recurso y en su evolución.

eTranslation, la nueva denominación de lo que se conocía hasta ahora como MT@EC, es el sistema de traducción automática (TA) de la Unión Europea que permite traducir automáticamente documentos y fragmentos de texto en un entorno de seguridad que garantiza la protección de los derechos de propiedad intelectual. El sistema incluye todos los idiomas oficiales de la Unión Europea y se espera que la traducción automática entre lenguas que presentan gran proximidad aumente la utilidad de los resultados de esta TA.

eTranslation proporciona un material de base para el trabajo de los traductores de la DGT y para los servicios linguiísticos de otras instituciones y órganos de la Unión Europea. Los resultados obtenidos con esta herramienta cuentan con la ventaja de que el sistema se construye sobre un amplio corpus de legislación europea y otros documentos de la Unión. Asimismo, las observaciones de los traductores se convierten en intervenciones específicas para mejorar la calidad.

Esta herramienta está a disposición de las administraciones públicas de los Estados miembros y constituye, además, la base del componente elemental que conforma la plataforma eTranslation, desarrollada con financiación del MCE (Mecanismo "Conectar Europa") entre 2015 y 2020 (véase https://ec.europa.eu/digital-single-market/en/connecting-europe-facility). El MCE forma parte del mercado único digital, una de las diez prioridades de la Comisión Juncker.

eTranslation - herramienta interna, pero también el punto de partida de la plataforma de traducción automática, componente elemental de gran importancia en el ecosistema de servicios públicos digitales del $\mathrm{MCE}$ - seguirá siendo un proyecto emblemático para la Comisión Europea.

\section{Siendo la calidad uno de los principios fundamentales, ¿cómo se controla la calidad de la traducción automática?}

La traducción automática en la DGT es, en cualquier caso, un recurso básico y auxiliar, integrado por completo en nuestras herramientas de TAO. Como consecuencia del riesgo de errores inherente, nuestros estándares de calidad establecen que los resultados obtenidos mediante traducción automática deben poseditarse y revisarse.

Por razones obvias de responsabilidad y seguridad jurídica, para nosotros la calidad es un factor de suma importancia. En línea con los principios definidos en el Marco de gestión de la calidad (Quality Management Framework) de la DGT, el equipo de gestión de calidad lo forman cuatro gestores, uno por cada una de las Direcciones (A, B, C y D) de traducción, que se reparten en dos sedes: Bruselas (Dirección A y Dirección D) y Luxemburgo (Dirección B y Dirección C). El Marco de gestión de la calidad en la DGT ofrece definiciones para los principios y conceptos clave en torno a la gestión de calidad y describe, además, la estructura del trabajo relacionado con esta actividad y sus principales participantes y procesos.

Las Directrices sobre calidad para la traducción (Translation Quality Guidelines) de la DGT sirven como guía para la traducción, el control de calidad y la evaluación de riesgos y, además, dividen los textos en cuatro categorías según su función. Nuestros coordinadores de 
calidad también han redactado documentos de referencia básicos sobre la traducción y revisión, así como sobre la evaluación y reducción de riesgos que conforman una versión breve de las directrices.

En paralelo al control de calidad interno, hemos elaborado las Directrices para la evaluación de traducciones externas (Guidelines for Evaluation of Outsourced Translations) de la DGT, en las que se describe el proceso de evaluación, calificación y control de calidad de este tipo de traducciones.

Para establecer referentes de calidad en cuanto a los contenidos, los departamentos lingüísticos de la DGT disponen de directrices y guías de estilo específicas para cada lengua. El Departamento de Lengua Española (DLE) ha elaborado la Guía del Departamento que, pese a necesitar algunas actualizaciones, proporciona información de gran utilidad tanto a nuestros traductores como a los traductores externos.

Los gestores de la calidad se reúnen semanalmente para coordinar y comentar la situación referente a las medidas en torno a la calidad, plantear cuestiones, llegar a acuerdos sobre líneas de acción y compartir información. En general, estas reuniones se celebran a través de MOVI o, para algunos temas concretos, por videoconferencia. También se organizan encuentros presenciales para que la comunicación sea más fluida.

Por otro lado, los gestores y los responsables de la calidad de los departamentos lingüísticos se reúnen dos veces al año y celebran, además, encuentros informales, de forma separada en Bruselas y Luxemburgo, para deliberar sobre temas pendientes y compartir información. Los gestores de la calidad organizan, una o dos veces al año, reuniones con los validadores y con los corresponsales externos de los departamentos lingüísticos para tratar aspectos relacionados con la externalización.

En nuestro Marco de gestión de la calidad, esta última se define como "el cumplimiento de las especificaciones o la adecuación a los objetivos". Este marco identifica los procesos principales para el aseguramiento de la calidad.

La DGT asigna tareas de traducción y revisión basándose en evaluaciones de riesgos en las que se tienen en cuenta la finalidad de los documentos y el perfil de competencias del personal. Los textos se agrupan en cuatro categorías en función de sus objetivos y los riesgos implicados:

1. Documentos legislativos.

2. Documentos normativos y administrativos.

3. Información para el público.

4. Documentos recibidos.

Todos los trabajos de traducción deben ajustarse a los principios generales y requisitos de calidad para la traducción profesional que establece la norma internacional ISO 17100.

\section{El uso masivo de la TA o de herramientas TAO iha supuesto un cambio de perfil en el traductor? ¿Podría señalar algunos elementos nuevos que a su juicio deberían incluirse en la formación del traductor para la UE?}


No cabe duda de que el entorno del traductor se ha digitalizado. Los llamados milennials o «nativos digitales» están familiarizados con la tecnología de forma natural, por lo que la competencia digital de traductores jóvenes no es un problema, ya que han recibido la formación necesaria en la universidad.

Como ya hemos señalado, aparte de las herramientas comerciales, la DGT ha desarrollado una serie de aplicaciones internas para gestionar la administración y el flujo de trabajo de las solicitudes de traducción y los documentos relacionados, así como para respaldar la labor de traductores y otras actividades. Por tanto, proporcionamos a los usuarios finales formación interna sobre procesos y herramientas como CatTool/Studio, Tradesk (translators' desktop), DGTVista, Mandesk, Poetry, Euramis, LegisWrite, E-Greffe, etc. Por ello, todo nuevo traductor o asistente que se incorpora a la DGT ha de seguir, como parte de su proceso de formación obligatoria, una formación sobre estas herramientas. Además de esta formación de base, disponemos, como cualquier otra gran organización, de nuestra propia estrategia de gestión del conocimiento.

En conclusión, los conocimientos básicos de informática no son suficientes para aquellos traductores que deseen sacar partido de las nuevas ofertas de trabajo relacionadas con el mercado de la traducción y la localización, como son las de gestor de proyectos, desarrollador de aplicaciones lingüísticas o especialista en localización. Las universidades han de ofrecer formación adecuada para cubrir estas necesidades.

\section{Siendo IATE una herramienta de larga tradición en la UE ¿podría dar una definición breve e indicar cómo ha evolucionado desde su creación?}

IATE es una base de datos dinámica diseñada para servir de ayuda a la redacción multilingüe de textos (y, más concretamente, de textos legislativos) de la Unión Europea. Su objetivo es proporcionar información relevante, fiable y de fácil acceso, que contenga un valor añadido obvio con respecto a la información que nos proporcionan otros recursos léxicos o documentales, como las memorias de traducción, archivos electrónicos y, en general, internet.

Esta base de datos debe gestionarse con rigor para poder integrarla a programas de ayuda a la traducción que pueden recuperar información de IATE, pero (a diferencia de lo que puede hacer el traductor) no detectar discrepancias entre las distintas versiones lingüísticas.

Nuestra base de datos terminológicos está integrándose progresivamente en nuestro entorno de herramientas de TAO. Nuestra unidad de terminología central prepara periódicamente extracciones de todo el contenido de IATE para su utilización directa en SDL Studio. Además, estamos desarrollando bases de datos específicas sobre ciertas materias, como economía y finanzas o migración, o sobre paquetes de documentos concretos, en los que la armonización a nivel terminológico es esencial desde el principio.

El grupo de trabajo IATE 2, creado por el grupo de gestión de IATE, está procediendo a cambiar la versión actual de la base y las interfaces para los usuarios por una solución más moderna y mejor adaptada a los métodos de trabajo de las instituciones que forman parte de IATE. En la práctica, estamos yendo más allá de una simple mejora en la introducción de datos, y estamos a punto de asistir al nacimiento de una nueva IATE, completamente rediseñada y con funciones adicionales: una pantalla para administrar tareas (que facilitará la integración de la gestión de proyectos terminológicos y del avance del trabajo en curso), un 
panel del usuario y más funciones de comunicación. Ahora se está probando la nueva versión de la interfaz de IATE y más adelante, seguramente a finales de este mismo año, se complete el proyecto IATE 2.

\section{Siendo la terminología el fundamento de IATE, ¿podrían indicarnos qué cambios se han producido en el tratamiento y uso de la misma en la última década?}

Hemos de recordar, en primer lugar, que la terminología multilingüe supone una gran contribución a la política de promoción del multilingüismo de la Unión Europea y a la transparencia de su legislación para todos los ciudadanos. En nuestra opinión, las palabras clave en relación a la evolución de la terminología son; autoridad, validación, interactividad, redes, datos enlazados, difusión y acceso al conocimiento.

La terminología en la red nos permite desarrollar todo el potencial de interactividad. Al ser una base pública, IATE proporciona a los traductores autónomos acceso a terminología validada y también permite a los terminólogos recibir comentarios de usuarios externos.

Los traductores buscan constantemente fuentes autorizadas, por lo que los terminólogos hemos de centrarnos en la validación y la calidad. Por este motivo, el Departamento de Lengua Española ha apoyado el proyecto Terminesp desde el primer momento y está implicado a fondo en su desarrollo. Esta plataforma nos brindará acceso a terminología validada $\mathrm{y}$, al mismo tiempo, facilitará la difusión de nuestros propios recursos terminológicos. Estamos sumergidos en un volumen gigantesco de información, pero lo importante no es la cantidad, sino la calidad. Idealmente, sería posible considerar incluso la participación directa de especialistas en el proceso de validación de términos o colecciones, lo que aumentaría la fiabilidad de nuestros datos terminológicos $\mathrm{y}$, por consiguiente, la coherencia de nuestras traducciones.

Por otro lado, las bases terminológicas se convertirán con el tiempo (de hecho, este proceso ya está en marcha) en repositorios de conocimiento en los que los términos pueden servir más bien como un índice o catálogo que facilite el acceso a una gran cantidad de recursos especializados. Pronto sabremos qué nos depara el futuro en este sentido.

7. Aparte de las TICS y los avances tecnológicos en la comunicación también se están produciendo otros cambios en las sociedades en su evolución hacia sociedades multiculturales y con los movimientos de población migrante y refugiados con sus lenguas y culturas, ¿de qué modo ha afectado o pueden afectar estos cambios en la política lingüística de la UE y concretamente en la DGT?

La Unión Europea se basa en el principio de «unidad en la diversidad»: diversidad de culturas, costumbres, creencias y lenguas. El artículo 22 de la Carta de los Derechos Fundamentales de la Unión Europea, proclamada el año 2000 y enmendada en 2007, exige que la Unión respete la diversidad cultural, religiosa y lingüística. El artículo 21 prohíbe toda forma de discriminación, entre las que se incluye la discriminación por razón de lengua. Este principio no solo se aplica a las lenguas oficiales de la Unión Europea, sino también a los numerosos idiomas regionales y minoritarios que utilizan determinados grupos de la población. El respecto a la diversidad lingüística es un valor fundamental en la Unión 
Europea, junto con el respeto al individuo, la apertura a otras culturas, la tolerancia y la aceptación del otro.

El Tratado de Funcionamiento de la Unión Europea (TFUE) garantiza las libertades y los derechos recogidos en la Carta de los Derechos Fundamentales de la Unión Europea, que pasa a ser jurídicamente vinculante.

A diferencia de otras organizaciones internacionales que trabajan, además, con un menor número de lenguas, las instituciones de la Unión Europea tienen mayor capacidad ejecutiva y, por ello, aprueban leyes que son directamente vinculantes para los ciudadanos de la Unión Europea. Por una simple cuestión de equidad y justicia, los ciudadanos y los tribunales europeos deben poder entender las leyes que han de acatar y hacer cumplir. La Comisión Europea debe comunicarse con los ciudadanos, los gobiernos nacionales, las administraciones públicas, las empresas y otras organizaciones a lo largo del territorio europeo en sus respectivos idiomas. La sociedad tiene derecho a saber qué se va a hacer en su nombre y ha de poder desempeñar un papel activo en estos procesos.

La base legal del multilingüismo en la Unión Europea se fundamenta en los artículos 20 y 24 del Tratado de Funcionamiento de la Unión Europea y en el Reglamento n. ${ }^{\circ} 1$ del Consejo de 1958, por el que se fija el régimen lingüístico de la Comunidad Económica Europea:

Las lenguas oficiales y las lenguas de trabajo de las instituciones de la Unión serán el alemán, el búlgaro, el castellano, el checo, el croata, el danés, el eslovaco, el esloveno, el estonio, el finés, el francés, el griego, el húngaro, el inglés, el irlandés, el italiano, el letón, el lituano, el maltés, el neerlandés, el polaco, el portugués, el rumano y el sueco.

Además de esta declaración oficial, la Unión Europea promueve activamente que sus ciudadanos aprendan otros idiomas, tanto para facilitar su movilidad profesional y personal dentro del mercado único como para promover un canal que permita establecer contactos interculturales y facilite la comprensión mutua. Aprender y hablar otros idiomas hace que nos abramos más a los demás, a sus culturas y opiniones, mejora nuestra capacidad cognitiva y fortalece, incluso, nuestra habilidad para usar nuestra lengua materna. Además, nos permite aprovechar al máximo las ventajas que nos ofrecen la libertad de trabajar o estudiar en otro Estado miembro de la Unión Europea o fuera de ella.

\section{8. ¿Creéis que hoy por hoy existe cierta sensibilización por parte de la EU y DGT hacia las lenguas no oficiales de la UE (p.e. árabe, chino, lenguas africanas) con un uso mayor que algunas de las lenguas oficiales?}

En nuestra Dirección General, se traduce cada año (principalmente en el Departamento inglés) un cierto volumen (reducido) de textos redactados en lenguas no oficiales de la Unión Europea. Nuestra Unidad de Comunicación podrá seguramente proporcionar estadísticas al respecto, si se hace una petición de forma oficial.

Tres de nuestros veinticuatro idiomas oficiales lo son también de las Naciones Unidas: inglés, francés y español. La DGT celebra reuniones periódicas con los servicios lingüísticos de las Naciones Unidas en el marco de la IAMLADP (Reunión Anual Internacional sobre Disposiciones en materia de Idiomas, Documentación y Publicaciones) y la JIAMCATT (Reunión Anual Internacional sobre Traducción y Terminología Asistidas por Ordenador) 
para incrementar los intercambios entre los servicios lingüísticos de organizaciones internacionales y organismos nacionales.

En 2010, durante la celebración del año Europeo de Lucha contra la Pobreza y la Exclusión Social, la DGT organizó un seminario sobre la Traducción y la Interpretación contra la Exclusión Social en Madrid, con el apoyo del equipo de la catedrática Carmen Valero Garcés (Universidad de Alcalá) y de la CEAR (Comisión Española de Ayuda al Refugiado). Las actas están disponibles en el siguiente enlace: https://cvc.cervantes.es/lengua/tices/.

Por otro lado, en lo que respecta al uso de idiomas en los tribunales, la Unión Europea publicó en 2010 la Directiva relativa al derecho a interpretación y a traducción en los procesos penales y la Comisión Europea apoyó la labor del Foro de Reflexión sobre el Multilingüismo y la Formación de Intérpretes, que sirvió para redactar el documento de base para la elaboración de la Directiva mencionada.

Más allá del marco legislativo, nuestra Dirección General siempre ha mostrado interés por la situación de la profesión de traductor en los Estados miembros. En España, a través de nuestra delegación en Madrid, organizamos y potenciamos las reuniones de la RITAP (Red de Intérpretes y Traductores de la Administración Pública) para la elaboración del Libro Blanco de la Traducción e Interpretación Institucional en España, que se publicó gracias al compromiso financiero de la Comisión Europea y del Ministerio de Asuntos Exteriores español.

\section{9. ¿Está preparada IATE para integrar o ampliarse a lenguas no-oficiales de mayor uso en la UE?}

No sería un problema, al menos desde un punto de vista técnico, ya que a IATE puede incorporarse contenido en todos los idiomas. Sin embargo, la cuestión está en si tenemos capacidad de gestionar contenidos en lenguas no oficiales de la Unión Europea para las que no disponemos de traductores o terminólogos. Debemos ser realistas y concentrarnos en nuestras prioridades y nuestro cometido, que ya es bastante complejo: gestionar una base de datos multilingüe y multitemática. Somos perfectamente conscientes de que no podemos abarcar todo (todos los términos de todos los ámbitos de especialidad) y no pretendemos, desde luego, convertirnos en el único recurso terminológico del mundo. La integración y la complementariedad de recursos terminológicos, a través de aplicaciones de datos enlazados, por ejemplo, podría ser la mejor solución a medio plazo.

\section{0. ¿Cómo puede contribuir IATE a difundir o ayudar en la consolidación de la Traducción e Interpretación en los Servicios Públicos (TISP)?}

Siempre estamos dispuestos a integrar recursos terminológicos fiables y validados, especialmente aquellos que añadan un valor a nuestro repertorio documental o fraseológico (memorias de traducción, textos alineados). La traducción y la interpretación en los servicios públicos proporcionan un buen número de recursos terminológicos, provenientes de situaciones comunicativas muy diversas y, por tanto, pueden resultar altamente relevantes para nuestro trabajo, que no se limita a la traducción legislativa.

En el mes de junio de 2017, la VII Jornada de Terminología y Traducción Institucional (un encuentro bienal que organiza el Departamento de Lengua Española (DLE) con la 
colaboración de la Representación de la Comisión en Madrid) se dedicó a la cooperación terminológica entre universidades españolas y la DGT. Presentamos un borrador del memorando de acuerdo a la CCDUTI (el organismo que reúne a todos los centros académicos de traducción e interpretación en España) para canalizar esta cooperación. Según este texto, las principales tareas y acciones que se deberían emprender son las siguientes:

- Por parte de la CCDUTI:

- elaborar una relación de centros académicos interesados en colaborar con el DLE;

- preparar un inventario de los trabajos terminológicos realizados por sus asociados que puedan interesar al DLE para su incorporación a la base de datos terminológicos IATE, plataforma pública de terminología europea;

- proporcionar formación adecuada de sus estudiantes a fin de capacitarlos, bajo supervisión del DLE, para la alimentación de la base IATE.

- Por parte del DLE:

- determinar los campos temáticos en los que sería deseable contar con la colaboración terminológica de los centros y departamentos asociados a la CCDUTI;

- facilitar la formación necesaria para trabajar en la base IATE a los centros de la CCDUTI interesados;

- revisar los contenidos terminológicos procedentes de la CCDUTI para adaptarlos a los criterios metodológicos de la base IATE;

- difundir (por ejemplo, en el boletín interinstitucional puntoycoma y en las redes en las que participa el Departamento), los trabajos terminológicos más destacados de los centros de la CCDUTI.

- De manera conjunta por parte de la CCDUTI y el DLE:

- estudiar las fórmulas concretas de cooperación (estancias en la DGT, trabajo a distancia, creación de espacios colaborativos mediante redes virtuales, etc.), así como los métodos de evaluación y seguimiento del avance de los proyectos de colaboración;

- difundir los frutos de la colaboración en foros y plataformas de iniciativas terminológicas de alcance más general (por ejemplo, en Terminesp) o en el marco de proyectos multilingües (como Realiter).

11. Siendo IATE un recurso abierto ¿cómo puede el ciudadano, investigador, o formador en las lenguas no-comunitarias contribuir al desarrollo y uso de IATE?

Como complemento a la respuesta anterior, la cooperación terminológica entre diferentes organizaciones es un proceso que ha de beneficiar a todas las partes interesadas, con el objetivo último de mejorar la difusión de los términos adecuados $\mathrm{y}$, por tanto, su validación como resultado de un proceso de aceptación general. Todos los participantes deberían tener un gran interés en implicar a socios más cualificados. 
12. ¿Alguna recomendación o consejo para los futuros traductores que ven en la UE (o en la DGT) una posibilidad de trabajo?

Además de las oportunidades laborales que ofrecen los procedimientos de selección organizados por las instituciones (véase la página web de EPSO, la Oficina Europea de Selección de Personal, https://epso.europa.eu/home_en), la externalización creciente de traducciones que se está produciendo tanto en la DGT como en otras instituciones ofrecerá, sin duda, nuevas posibilidades a los traductores autónomos.

La traducción es una profesión muy exigente, en constante cambio. Un traductor debe ser curioso por naturaleza. Por ello, recomendamos a nuestros jóvenes colegas que exploren todas las posibilidades de sus horizontes académicos y profesionales, sin limitarse a una institución específica. 